\title{
Etiology of Cesarean Uterine Scar Defect (Niche): Detailed Critical Analysis of Hypotheses and Prevention Strategies and Peritoneal Closure Debate
}

\author{
Shashikant L. Sholapurkar
}

\begin{abstract}
There is an increasing incidence of cesarean scar (CS) defect/niche and its sequelae, probably not entirely explained by better diagnosis or rising cesarean rate. Discussion of possible etiological factors has received scant attention but would be important to formulate preventive strategies. Meaningful informative studies on long-term sequelae of cesarean section are very difficult and none are available for causation of CS defect. Hence, it is crucial to identify key areas in etiology of CS defect for focused research. This practical review proposes an "ischemia and mal-apposition hypothesis for CS niche", stating that the surgical technique of uterine incision closure is the most important determinant of CS defect formation. Other factors such as cervical location incision, adhesion formation and patient specific factors seem far less important in etiology. Rather than the headline theme of "single versus double-layer closure of uterus", the finer details of surgical technique which achieve good apposition without inducing tissue ischemia seem more important. Different techniques are discussed and it is proposed that continuous, non-locking absorbable sutures in two layers, without including much of decidua and without undue tight (constricting/devasculaizing) pulling of sutures are likely to result in good healing of uterine scar. Single-layer technique may be best reserved for thin myometrial edges especially during repeat cesareans. Adhesions between uterine isthmus and bladder/abdominal wall seem common associations but not causative for CS niche. It would be desirable to prove these surgical principles by good quality prospective randomized "quantitative" studies but the wait may be very long and this should not hinder the adoption of good surgical principles. Science is much cognitive and not just empirical. To consider a related example, the current recommendation of non-suturing of peritoneal layers during cesarean is mistakenly based on short-term irrelevant surrogate outcomes like analgesic requirements and time-saving, many of which have been already disproven. Evidence is presented recommending simple quick techniques of peritoneal closure to prevent adhesions. More analytical debate in surgical techniques is needed to inspire engaged, critical and insightful practitioners rather than unquestioning
\end{abstract}

Manuscript submitted November 13, 2017, accepted January 8, 2018

Department of Obstetrics and Gynaecology, Royal United Hospital, Combe Park, Bath, BA1 3NG, UK. Email: s.sholapurkar@nhs.net

doi: https://doi.org/10.14740/jocmr3271w dependence on weak evidence/guidance.

Keywords: Cesarean scar defect; Cesarean scar niche; Uterine incision closure; Cesarean single layer closure; Cesarean double layer closure; Etiology of cesarean scar defect; Peritoneal closure during cesarean; Adhesion formation after cesarean

\section{Introduction}

Cesarean section is by far the commonest major operation worldwide [1] with progressively rising incidence and consequently any long-term adverse sequelae assume increasing importance. In the last two decades, there has been increasing awareness of long-term gynecological problems after cesarean like chronic pelvic pain, dyspareunia, dysmenorrhea, postmenstrual spotting and even infertility [2-4]. Moreover, long-term obstetric sequelae seem to be on the rise in the form of a spectrum of disorders starting with cesarean scar (CS) ectopic pregnancy, increased incidence of placenta previa, varying degrees of adherent anterior placenta praevia including placenta accreta associated with major maternal morbidity and even mortality [5-7]. Interestingly, a very rare gynecological complication of massive uterine hemorrhage during menstruation due to CS defect requiring emergency uterine artery embolization followed by laparoscopic repair of defect has been described [8]. In addition, a possibility needs to be entertained that at the extreme end of obstetric sequelae, there may be very rare cases of profuse late ( $4-8$ weeks) postpartum hemorrhage (PPH) sometimes leading to hysterectomy (unpublished clinical correspondence) where diagnosis of CS defect (nonunion) is overlooked or such cases go unreported/unpublished. Many senior obstetricians express a subjective perception of a disproportionate rise in incidence of cesarean uterine scar (CS) niche not entirely explained by increased diagnosis and cesarean rate. In the last decade, there has been upsurge in the literature about complications of CS defect and treatment modalities. However, not much attention has been paid to the possible etiology of CS defects which would help to reduce the future incidence of CS defects and long-term complications. The author was an early protagonist to propose that surgical technique of uterine incision closure seems the most important factor in poor healing of CS scar leading to CS defect $[9,10]$. 
Hence, the formation of CS defect and its long-term sequelae should not be considered inevitable $[9,10]$.

The term CS niche (defect) describes the presence of a hypoechoic area within the myometrium in the isthmus (lower uterine segment) with discontinuation of myometrium at the site of previous CS $[3,11]$. A niche has been described as the indentation of myometrium of at least $2 \mathrm{~mm}$. Large niches are uncommon with reported varying incidence of $11-45 \%$ depending on the definition used. A significant niche is defined as involving a depth of at least $50-80 \%$ of anterior myometrium or the remaining myometrial thickness less than $2.2 \mathrm{~mm}$ when evaluated by transvaginal ultrasound scanning (TVS) [3]. Small niches may indeed be quite common but would be clinically unimportant. The large niches are most likely to give rise to long-term sequelae, hence the focus of research and discussion should be the causation of large niches and prevention strategies. Recently, there has been a detailed review/clinical opinion paper with description of numerous possible etiological factors involved in causation of CS defect [12]. This comprehensive description is of course very useful and forms a good basis for downstream debate and analysis as attempted in this paper. It has also been proposed that randomized controlled trial (RCTs) should be designed to study many of these factors [12]. However, this seems a Herculean and impossible task. Historically, there has been little success in designing informative RCTs/studies comparing different CS techniques and previous studies have struggled to resolve even simple controversies like peritoneal closure. Good quality well-designed studies are of course very important in medical field. These do generate a lot of numerical data but such a quantitative approach is not guaranteed to be successful unless the underlying starting hypotheses are meaningful or valid. Science is (much) cognitive, not (just) empirical [13]. "Numbers gathered without sound knowledge almost always remain just numbers. The route from hypothesis (theories) to law (framework/system) to measurement (studies/trials) can almost never be travelled backwards" - Thomas Kuhn, 1961 [13]. As per the "IDEAL" (Idea, Development, Exploration, Assessment, and Long-term study) framework for new concepts [14], the knowledge about etiology of CS defects seems to be at the first two stages. At the same time, it seems impractical (probably impossible) and wasteful to examine/study large number of theoretical etiological factors, many of minor importance, by good quality studies. Hence, this clinical review critically analyzes all the previously suggested etiological hypotheses of CS niche formation $[9,10,12]$, in order to identify the key areas where clinical discussion and research (especially RCTs) should focus. This analysis is not an "arm-chair theorist's approach" but quite the opposite. The analysis is based on the long ongoing hands-on clinical experience, interest and observations by the author and colleagues worldwide and on the limited number of studies available. The article also discusses what answers could be practically expected from systematic studies and RCTs. Moreover, it also explores how good surgical practice can be discussed, developed and promoted by critical abductive thinking, while we wait for the result of RCTs as the wait may be very long indeed or even indefinite. A related subject of the controversy surrounding peritoneal closure during cesarean is also discussed to highlight some of these points.

\section{Ischemia and Mal-Apposition Hypothesis for CS Niche (Techniques of Closure of the Uterine Incision)}

This review proposes a leading hypothesis that ischemic necrosis of some of the myometrium in the uterine incision edges (due to very tight and/or locking suturing) is a more important cause of development of CS defect. The subject of ischemic impairment has received hardly any attention in the past. Defective/ inappropriate apposition of myometrial layers in the form of interposing excessive decidua and inclusion of very wide bites of adjoining normal myometrium also makes a contribution. This hypothesis derives from a common observation (in addition to reasons discussed below) during repeat cesareans when a large area over the lower uterine segment is found to be very thin and there is remarkable scanty bleeding on incising such a lower uterine segment. The explanation seems that a thin area of muscle to muscle union is formed due to ischemia-malapposition during healing of the first cesarean. When the lower uterine segment unfolds during subsequent pregnancy, this thin band of myometrium gets stretched far more than the surrounding normal myometrium. This hypothesis is described in details below.

This paper argues that the surgical technique of the closure of uterine incision seems the most important factor in the development of CS defect/niche and long-term sequelae. The rubric has generally bogged down to a single versus double-layer closure (and resultant good or deficient apposition/alignment). Such dichotomous headline distinction permits RCTs but has failed to resolve the controversy. The crucial factor is not the appellation (name) of the method but the finer details of the constituent surgical techniques with their advantages and disadvantages, either of which could be maximized/diminished whichever headline method is used. The double-layer closure (Fig. 1a) was established because it has the potential advantage of better apposition of thick uterine muscle edges (especially during the first cesarean and then subsequent ones). The hardly discussed rationale for the single-layer closure seems the potential for less devascularization/ischemia of myometrial edges which will influence the thickness of the muscle-tomuscle healing. Thus, it behoves that achieving good apposition of myometrial edges without devascularization/ischemia is the crucial factor rather than the simplistic concept of single versus double-layer closure. Use of synthetic absorbable sutures like polyglycolic acid (Vicryl, Ethicon Ltd) with tensile strength for 2 - 3 weeks is now commonplace. With these modern tissue-friendly sutures, the amount/type of suture material is unlikely to be of much importance.

\section{Non-closure of deeper myometrial layers}

This does not seem an important cause. In Netherlands, in most of the cohort studies, $95 \%$ of the cases had a single layer closure (Fig. 1b) without peritoneal closure and in only half of the cases, non-inclusion or avoiding of substantial endometrium/ decidua was practiced [12], but CS niches do occur. A hypothesis has been proposed that during closure of uterine incision, an unintentional omission to include deeper myometrial lay- 


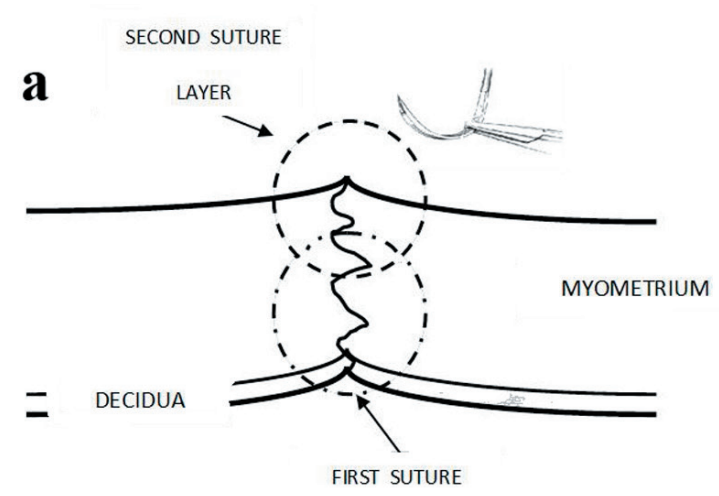

LAYER

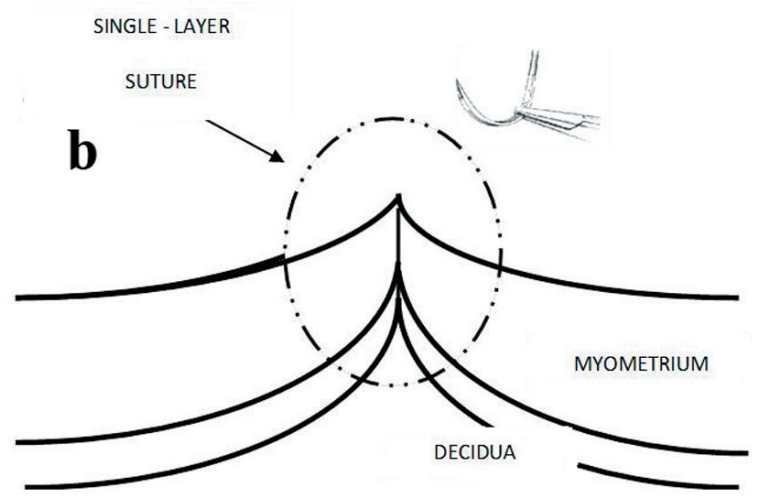

SECOND SUTURE

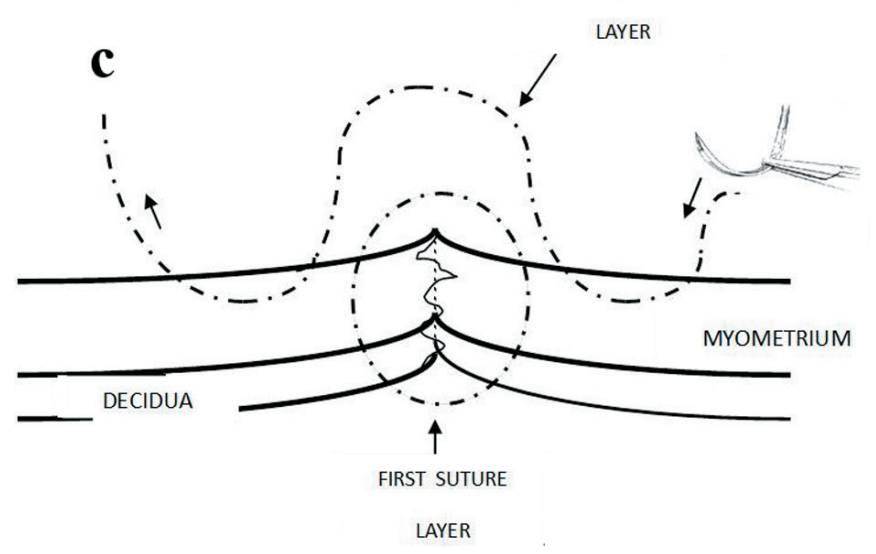

Figure 1. (a) Recommended traditional judicious double layer closure with the first continuous non-locking suture to include minimal decidua $(<5 \mathrm{~mm})$ and about two-thirds of inner myometrium; and second nonlocking suture taking upper half of myometrium would correct eversion of myometrial edges. This used to be the long-standing practice in UK more than a decade ago. Care should be taken not to make the edges of the incision ischemic. (b) One-layer closure could interpose decidua in between inner myometrium and the superficial myometrial edges can often be seen to be everted (not in good apposition). (c) The current popular technique in UK. The transverse myometrial bites of second layer are taken with the needle travelling back and forth on either side of incision which seem partly akin to "figure-of-eight" haemostatic/devascularizing sutures. It is easy to be paradoxically reassured by the apparent (excessive) apposition and sense of security derived from very tight sutures. Ischemic necrosis is likely to be causative in CS defect. ers could explain subsequent disruption of inner myometrial layer and development of niche [12]. Additional potential factors have been proposed such as superficial closure due to nonperpendicular (tangential) endometrial saving) [12]. However, an almost universal observation in the UK has been that it is quite difficult to avoid inclusion of at least some decidual layer (despite special attention and effort) in the closure of uterine incision. Hence, the omission of closure of the deeper layers of myometrium is virtually never observed being practiced in the UK (Fig. 1a, b, c). Hence, inclusion of more $(\geq 0.5 \mathrm{~cm})$ than less of decidua is almost a universal practice during the closure of uterine incision rather than missing the deeper layers of myometrium. Thus, the non-closure of deeper myometrial layer could not be the common cause of CS niche. On the other hand, the inclusion of excessive decidua interposed between the deeper layers of myometrium may contribute to deficient fusion of inner myometrium leading to a niche (Fig. $1 b, c)$. Moreover, with a single layer closure especially without endometrium saving technique, it can be practically observed that the cut myometrial edges evert in between the suture bites, which may be indicative of suboptimal apposition.

\section{Mal-alignment versus ischemia of myometrium}

More importantly, most of the discussion about the hypotheses of CS niche formation in past seems to be focussed on deficient alignment (apposition) of myometrial tissue, but there has been hardly any attention paid to ischemia of tissues or preservation of vascularity of the myometrial edges sutured together. This is particularly surprising given that a number of published case reports of uterine scar dehiscence in the puerperium with massive late PPH predominantly show ischemic necrosis of the uterine scar (hence devascularization) in most cases [15-19]. This also raises a possibility whether lesser degrees of scar dehiscence (smaller complete or large incomplete defects particularly following repeat cesareans) could be the cause of very rare cases of late ( 4 - 6 weeks) heavy $\mathrm{PPH}$, such cases going undiagnosed or unreported. This review proposes that in addition to good apposition of the myometrial layers, it is equally important to prevent devascularization of the apposed edges; and aims to increase the awareness in this regard. The current commonly practiced technique in the UK (by peer imitation) generally involves quite tight first suture layer including entire thickness of myometrium and substantial decidua (Fig. 1c). While inserting the second layer of continuous suture, it has become a popular practice (seemingly without any cogent basis) to take transverse back and forth bites of intact myometrium almost $1 \mathrm{~cm}$ away from the incision and overlap these over the first layer which requires quite tight pulling of sutures. Could ischemia and necrosis (rather than lack of apposition) of some of the myometrial tissue ensue as a result and then contribute to CS defects? The resultant inflammatory reaction from ischemia/necrosis would also explain the associated (but not causative) fibrosis at the scar leading to dense adhesions of uterine isthmus to bladder and/or abdominal wall with increased retroversion of uterine body often seen in cases with CS defect.

The author has been called to assist with a case where the 
lower uterine segment had become extremely thin over a wide area following only one previous cesarean. The obstetrician had placed a tight transverse second layer of sutures during the second cesarean which simply cut through the thin myometrium, creating a transverse full thickness tear below and parallel to the entire length of uterine incision. This should raise questions about the practice of inserting tight transverse sutures during the first as well as subsequent cesareans.

There are of course a few more factors to be considered which influence the scar healing and adhesion formation, viz. inflammation due to infection, inadequate hemostasis, improper tissue manipulation and surgical trauma. Inadequate hemostasis has not been observed to be common at all; in fact rigorous hemostasis is the norm. Infection of the uterine CS is also very uncommon in developed countries. Gentle tissue handling needs to be taught more often. These factors may play a very minor role in CS defect causation.

\section{Recommended technique of uterine incision closure}

The technique demonstrated in Figure 1a has several theoretical and practical advantages to be recommended. Sumigama et al (2014) in a small retrospective study suggested that interrupted absorbable sutures for the first layer of myometrial closure had significantly lower incidence of placenta previa accreta [20]. They quite rightly described this as a small preliminary study leading to only weak recommendation. The number of cases included was very small especially when locking versus non-locking of continuous suture was concerned. They also highlighted several other confounding factors [20]. The interrupted suturing technique of uterine incision is not much used in Europe or America, because it is substantially more time consuming and impractical with the resultant delay in hemostasis. Moreover, continuous non-locking sutures which are not too tight are almost as good as interrupted sutures in preserving vascularity of the sutured tissues. At the same time, it seems equally important to avoid tight and/or locking sutures during the first as well as the second layer of sutures. The two-layer technique seems beneficial if it improves apposition of the myometrial edges which is commonly the case unless the lower segment has become very thin from previous cesareans. However, caution should be taken not to include too much surrounding myometrium with strangulating transverse, tight or locking sutures. Any persistent bleeding points are best controlled with separate simple or figure-of-eight fine (1/0 or 2/0) absorbable suture.

Three other hypotheses have been described in literature [12] which are not favored by this review and are mentioned below for the sake of debate and completeness.

\section{Hypothesis of Cervical Location of Cesarean Incision Leading to Niche}

The current paper does not support this proposition as a significant etiological factor. It was originally proposed by Vervoort et al as the first hypothesis [12]. It is unclear whether they intend it to be the leading or most important hypothesis. They propose that the thick mucoid brown discharge and a frequent finding in large niches of mucus accumulation and formation of large retention cysts supports this particular hypothesis [12]. Although it is an interesting observation, this hypothesis seems to lack broader base from direct practice observation and consideration of actual techniques of placing the cesarean uterine incision. Moreover, in the UK most scar defects on ultrasound are in the isthmic region or lower part of uterus. Placement of uterine incision in the cervix would be very rare in the UK and indeed technically quite difficult. Even in the second stage cesarean sections, this is rare as it would involve reflecting the bladder to a significant extent and placing the incision well below the reflection of bladder from the isthmic part of uterus. Based on the concern of placing the incision on the cervix, Vervoort et al question the practice of incising the loose peritoneal layer on the lower uterine segment to reflect the bladder down presumably even during the first cesarean or elective cesareans [12]. The purpose behind this age old traditional practice has been to reflect the bladder out of the harm's way in case there is downward tear in the lower uterine segment especially in advanced labor. The other purpose was to be able to cover the uterine incision with the loose peritoneum mobilized in this process. The pros and cons of closure of uterovesical peritoneum are discussed in details later in this review. Even though the bladder is separated from the lower uterine segment to some extent, the uterine incision is almost always placed just below the upper peritoneal reflection on the lower uterine segment generally above the original bladder attachment, thus very unlikely to be over the cervix. Secondly, after delivery of the baby, the lower flap of the uterine incision retracts and bladder can become quite close to the lower myometrial edge which could potentially interfere with suturing unless the bladder has been displaced away downwards beforehand. Hence, there seems good merit in the practice of incising the uterovesical pouch of peritoneum and reflecting the bladder down to a modest degree. More evidence (even observational) and awareness with good retrospective cohort studies is required regarding cervical location of uterine incision as a significant cause for CS defect. However, intentionally designed prospective studies do not seem feasible or practical in this regard for obvious reasons.

\section{Hypothesis That Dense Adhesions Following Some Surgical Techniques May Induce Niche Formation}

This analytic review does not favor this hypothesis. Vervoort et al present a very interesting and probably an important observation in patients having laparoscopic repair of large symptomatic niches [12]. This observation is that often these patients have dense adhesions anteriorly at the site of CS at the isthmic level and often the uterus is retroflexed. Moreover, the bladder can be adherent over the isthmus with peritoneum quite puckered. In some cases, the lower part of the uterus is adherent to the anterior abdominal wall. Vervoort et al show a diagrammatic representation to propose that the contraction of adhesions between the uterine scar and the abdominal wall would retrovert the uterus and also exert counteracting forces 


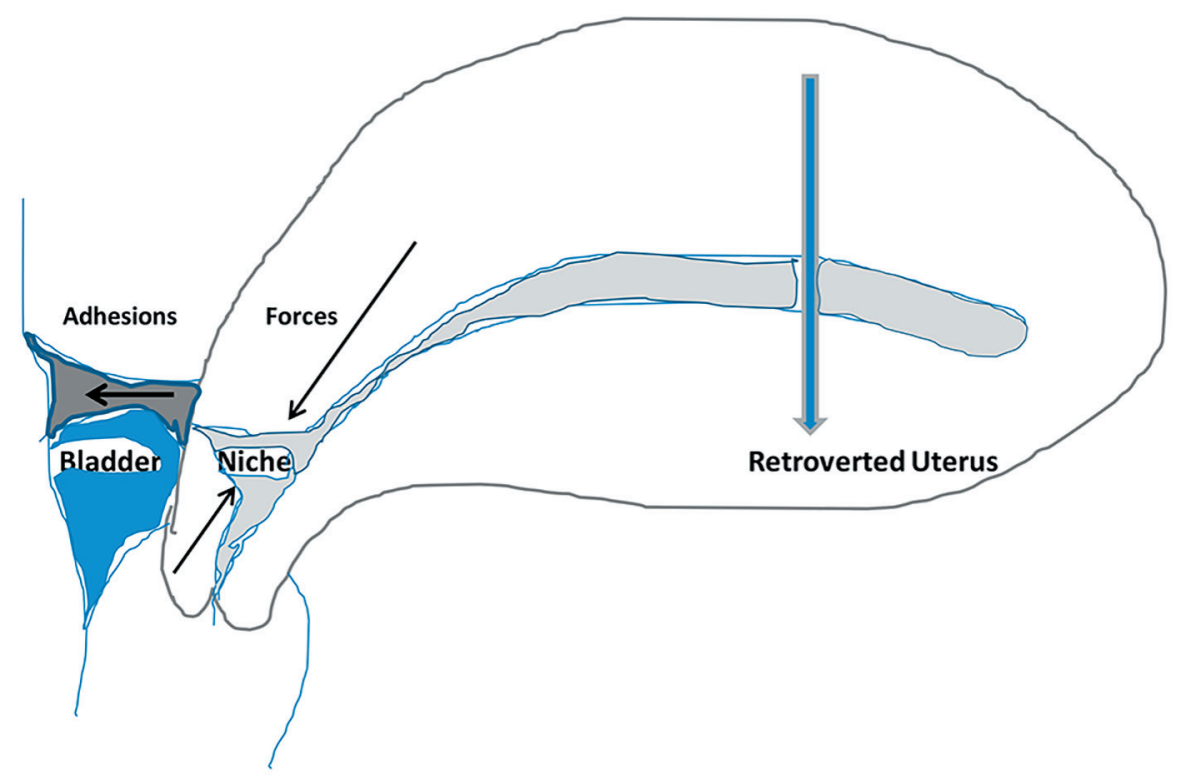

Figure 2. Schematic drawing of distorting forces (arrows) created by formation and retraction of adhesions between uterine isthmus (cesarean scar) and anterior abdominal wall combined with retroversion of uterus. The drawing illustrates that these forces do not seem to facilitate the formation (opening) of the CS niche. Hence, anterior adhesions are unlikely to be causative in formation of CS niche but are just associations.

on the uterine scar leading to a niche formation [12]. However, based on a closer analysis of that diagrammatic representation, it could be argued that the pull of adhesions could in fact exert forces in the opposite direction than shown thus opposing the tendency to form a niche. The more realistic forces from the contraction of adhesions and retroversion are shown in Figure 2. Particularly when the uterine body and fundus falls backwards, any distorting forces would act to close any scar defect rather than opening it (Fig. 2). Thus, there are rational reasons to question this hypothesis that the anterior adhesions are causative of the CS defect. Nevertheless, this observation by Vervoort et al [12] itself seems very appropriate in that some particular surgical techniques may produce CS niche on one hand and adhesions on the other hand. This review proposes that the ischemia and necrosis of some of the myometrium as a result of excessively tight suboptimal suturing technique could lead to $\mathrm{CS}$ niche and also inflammatory reaction resulting in the formation of adhesions between CS and anterior abdominal wall. In addition, non-closure of uterovesical pouch of peritoneum (combined with non-closure of parietal peritoneum) would lead to denuded and inflamed, ischemic or necrotic uterine area of CS in contact with peritoneum-denuded area of anterior abdominal wall leading to dense adhesions in some cases. The resultant pulling of the uterine isthmus towards anterior abdominal wall by adhesions can explain increased incidence of uterine retroversion [12].

\section{Hypothesis of Patient Specific Factors Impairing Uterine Wound Healing}

A few factors like genetic predisposition, obesity, preeclamp- sia/hypertension, etc. have been suggested [12]. However, there is little evidence to suggest that some patients could be more prone to form uterine scar defects. Any such influence would be very minor and mostly irremediable any way. Moreover, it would not be possible to design good quality studies including enough number of subjects to confirm or rule out these patient factors. Hence, this will remain simply a hypothesis of no practical importance mentioned here just to comment on the theoretical etiological list. It would be important to concentrate research efforts and limited resources on the important factor of uterine incision closure techniques.

\section{Debate About Peritoneal Closure During Cesarean}

This debate seems a major example of how a controversy is generated and then left unresolved by a simplistic and flawed numerical or quantitative approach to a surgical practice. It was a long-standing default position to approximate/suture uterovesical and abdominal (parietal) peritoneum involving a fairly easy technique of suturing with a fine $1 / 0$ or $2 / 0$ absorbable continuous non-locking suture. Unfortunately, long-term studies with big enough number of patients (and low drop-out rate) are very difficult to conduct and very few are available. It seems reasonable to argue that the default position of closing peritoneal layers should have been discontinued only if it could be proven by well-conducted long-term studies that the closing peritoneal layers do not reduce the adhesion formation or indeed increase it. Alternatively, non-suturing of peritoneum could be justified as a part of well-designed prospective research study. However, some studies focused on short-term 
outcomes in the form of irrelevant surrogate measures such as analgesic requirements, febrile episodes and shortening of operative time by about $5 \mathrm{~min}$, etc. and early results were utilized to recommend non-closure of peritoneum [9, 21]. Moreover, to begin with these conclusions were derived by rearward opportunistic trolling of data and applying statistical measures of significance (a well-recognized fallacious approach) to unrelated outcomes with no good causal relationship to the surgical technique in question. Some studies purely by chance showed statistically significant increase in these irrelevant surrogate short-term outcomes and many guidelines advocated non-closure of peritoneal layers [9, 21]. Such a convenient advice of course did prove very popular and this "meme" quickly became very widely adopted and even today remains very difficult to shake off. Evidence-based medicine is distorted by such flawed studies with numerical rhetoric because of the apparent and often deceptive "certainty" engendered by the "numbers" and flawed statistical reasoning presented. The combination of irrelevant outcome measures and total lack of "consilience" make the recommendation of non-closure premature and scientifically dubious. There are already better studies available which have refuted these irrelevant short-term drawbacks any way $[22,23]$. In addition, a small meta-analysis including 249 women [24] and another much larger systematic review by Shi et al [25] including 4,423 women, showed that the closure of visceral and parietal layers of the peritoneum significantly reduced formation all grades of adhesions especially the severe ones. Another small trial [26] did not show significant difference in adhesion formation but verification and other biases cannot be ruled out [12]. More importantly, there are case reports and case series reported [27] where dense adhesions between uterine isthmus and anterior abdominal wall give rise to long-term symptoms like pelvic pain, dyspareunia and even infertility, sometimes requiring operative intervention to release the adhesions. Moreover, the surgical difficulty during repeat cesarean because of extensive adhesions obliterating uterovesical pouch is being increasingly encountered. The non-closure of peritoneum has been considered most likely cause for these adhesions $[9,21,27]$. It can be causative in two ways. Firstly the heavy postpartum uterus can immediately drop low in pelvis and the lower flap of uterovesical peritoneum can rise leading to adhesion of bladder higher up on the lower uterine segment. Alternatively, the wide raw peritoneum-denuded areas on the lower uterine segment and the anterior abdominal wall can remain in contact with formation of adhesions varying from mild to dense and extensive [9]. The closure of the peritoneal layers would maintain the original isolation function of the peritoneum avoiding direct contact of raw areas. The author has personally operated on two such symptomatic cases despite his limited benign gynecological surgical practice. These two anonymized cases are described below.

\section{Case 1}

A woman in her 30s was on the elective gynecological theater list of this author for diagnostic laparoscopy. Ever since having a cesarean section about 2 years previously, this lady was unable to have intercourse because of very significant deep dyspareunia. Bimanual pelvic examination revealed cervix to be pulled anteriorly with cervical excitation pain. Laparoscopy revealed a $5-\mathrm{cm}$ thick and $5-6 \mathrm{~cm}$ long band of firm fibrotic adhesions between uterine CS (isthmus) and lower anterior abdominal wall. It seemed apparent that any movement of cervix with vaginal manipulation would stretch these adhesions and could account for pain. These adhesions were easily divided with diathermy scissors freeing the cervix and uterus from the abdominal wall. Patient's dyspareunia completely resolved and she did not require any further intervention.

\section{Case 2}

A woman in mid-20s admitted herself through casualty for constant lower abdominal pain. She was transferred to gynecology ward because her pain had started 6 months ago following her first cesarean section. She was also unable to have intercourse because of deep seated pain. She had seen her general practitioner a number of times with this complaint who apart from reassurance and analgesics could not offer much help. Ultimately, she decided to present herself to casualty. Abdominal examination revealed well healed Pfannensteil scar and pelvic examination revealed anterior tenderness which was thought to be non-specific and "functional". Pelvic ultrasound scan was normal. However, the patient refused to be discharged home and the author had to perform a diagnostic laparoscopy expecting to confirm normal findings. Surprisingly, a wide firm band of fibrotic adhesions was seen between uterine isthmus and lower abdominal wall. This was uneventfully divided with diathermy scissors. Patient was discharged home a few hours later and reported resolution of her constant lower abdominal pain as well as dyspareunia on follow-up.

\section{Why and How to Suture Peritoneum}

Most experienced obstetricians will concur that these relatively rare cases extensive adhesions of uterine isthmus to anterior abdominal wall will not occur if peritoneal layers are closed $[21,27]$. There seems to be a belief that objective proof of this observation must be offered by quantitative studies with statistical analysis. This is not necessarily so. Some observations can be strong enough that they do not necessarily need statistical proof, sometime referred to as the "IOT test" (interocular trauma test - the conclusions hit you in the middle of the eyes) $[28,29]$. Indeed it may not be possible to prove this by quantitative studies because of the methodological problems and confounding factors inherent. But insisting on statistical proof seems misunderstanding of evidence-based medicine. The suturing of uterovesical peritoneum could be considered a default position to be proved wrong. Once the widespread adhesions have formed it becomes near impossible to restore the anatomical peritoneal coverings over the lower uterine segment during the repeat cesareans. The author has modified his technique of uterovesical peritoneal closure to a single stitch in the middle with four bites (Fig. 3a, b). This often brings the peritoneal 

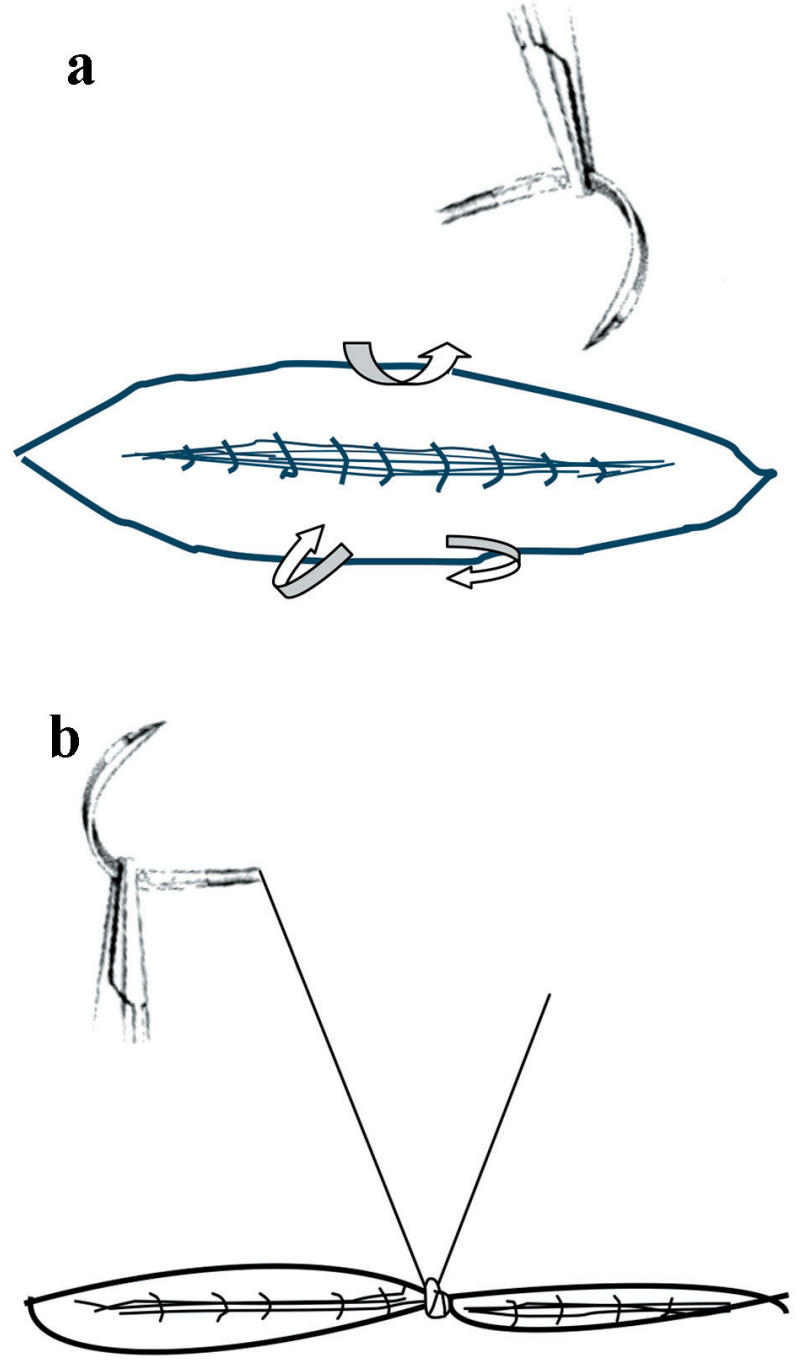

Figure 3. (a) Schematic drawing of a simple technique of single stitch closure of uterovesical folds of peritoneum during cesarean. (b) The result of single stitch closure of peritoneum which covers the uterine scar fairly well and dictates the normal anatomical healing thus preserving the deep uterovesical pouch. Occasionally an extra stitch may be required.

flaps close enough to prevent adhesions and maintains bladder low down in relation to the lower uterine segment. Occasionally one or two additional interrupted sutures may be required if apposition is not enough. Many of author's colleagues have enthusiastically adopted the technique after witnessing it. Suturing of abdominal (parietal) peritoneum would be ideal but requires more care to be brought together without tension so that stitches should not cut through to create gaps. Not suturing parietal peritoneum can induce omental adhesions which are generally thought to be of no consequence but it may be ideal to prevent them. Bowel adhesions (in the absence of bowel inflammation) do not occur because of peristaltic movement of bowel loops. Thus, closing/suturing of uterovesical peritoneum seems more important than parietal peritoneum, a matter generally not deliberated.

\section{Conclusions}

The incidence of cesarean uterine scar defect is increasing together with the resultant long-term sequelae. The surgical techniques of uterine incision closure seem to be the most important determinant in causation of CS defect or niche. The cut myometrial edges (unless very thin from previous cesareans) may be best sutured in two layers of non-locking continuous sutures without undue tightness and minimal inclusion of decidua (Fig. 1a). This would achieve best apposition without devascularization of myometrial edges. Ischemic necrosis of the some of the myometrial tissue best explains the formation of CS defect and accompanying scarring and adhesions. Closure of uterovesical pouch of peritoneum with very simple technique of just one or two interrupted sutures has the advantage of further reducing the adhesion formation. It would be ideal/desirable to prove these good surgical principles by prospective randomized studies which is a very difficult task and could take a very long time, but this need not hinder the adoption of rational practice based on good surgical principles.

\section{Acknowledgments}

The author would like to express acknowledgement and indebtedness to all previous authors and experts especially Vervoort et al [12] for their pioneering work in the management of cesarean scar defect. This review borrows from previous literature but has a different emphasis, content and conclusions. The author is also grateful to his Institute and its Library staff for providing easy and prompt access to the published literature. The concepts presented are opinion only.

\section{Grant Support}

No financial support received.

\section{Conflict of Interest}

The author has no conflict of interest to declare.

\section{References}

1. Mathai M, Hofmeyr GJ, Mathai NE. Abdominal surgical incisions for caesarean section. Cochrane Database Syst Rev. 2013;(5):CD004453.

2. Wang CB, Chiu WW, Lee CY, Sun YL, Lin YH, Tseng CJ. Cesarean scar defect: correlation between Cesarean section number, defect size, clinical symptoms and uterine position. Ultrasound Obstet Gynecol. 2009;34(1):8589.

3. Bij de Vaate AJ, Brolmann HA, van der Voet LF, van der 
Slikke JW, Veersema S, Huirne JA. Ultrasound evaluation of the Cesarean scar: relation between a niche and postmenstrual spotting. Ultrasound Obstet Gynecol. 2011;37(1):93-99.

4. van der Voet LF, Bij de Vaate AM, Veersema S, Brolmann HA, Huirne JA. Long-term complications of caesarean section. The niche in the scar: a prospective cohort study on niche prevalence and its relation to abnormal uterine bleeding. BJOG. 2014;121(2):236-244.

5. Clark SL, Koonings PP, Phelan JP. Placenta previa/accreta and prior caesarean section. Obstet Gynecol. 1985;66:8992.

6. Timor-Tritsch IE, Monteagudo A, Santos R, Tsymbal T, Pineda G, Arslan AA. The diagnosis, treatment, and follow-up of cesarean scar pregnancy. Am J Obstet Gynecol. 2012;207(1):44 e41-13.

7. Naji O, Wynants L, Smith A, Abdallah Y, Stalder C, Sayasneh A, McIndoe A, et al. Predicting successful vaginal birth after Cesarean section using a model based on Cesarean scar features examined by transvaginal sonography. Ultrasound Obstet Gynecol. 2013;41(6):672-678.

8. Shao MJ, Hu M. A growing concern: cesarean scar defect and massive uterine bleeding. J Clin Case Rep. 2015;5(9):599-600.

9. Sholapurkar SL. Increased incidence of placenta praevia and accreta with previous caesareans - a hypothesis for causation. J Obstet Gynaecol. 2013;33(8):806-809.

10. Sholapurkar SL. Surgical techniques at caesarean might modify placenta accreta risk. BJOG. 2015;122(1):143.

11. Naji O, Abdallah Y, Bij De Vaate AJ, Smith A, Pexsters A, Stalder C, McIndoe A, et al. Standardized approach for imaging and measuring Cesarean section scars using ultrasonography. Ultrasound Obstet Gynecol. 2012;39(3):252-259.

12. Vervoort AJ, Uittenbogaard LB, Hehenkamp WJ, Brolmann HA, Mol BW, Huirne JA. Why do niches develop in Caesarean uterine scars? Hypotheses on the aetiology of niche development. Hum Reprod. 2015;30(12):26952702.

13. Bookstein FL. "Numbers and reasons" - core elements of numerical inference techniques today, with implications for the rhetoric of LASR presentations. 2010. https:// www1.maths.leeds.ac.uk/statistics/workshop/lasr2010/ proceedings/L2010-05.pdf.

14. Nikkels C, Vervoort A, Mol BW, Hehenkamp WJK, Huirne JAF, Brolmann HAM. IDEAL framework in surgical innovation applied on laparoscopic niche repair. Eur J Obstet Gynecol Reprod Biol. 2017;215:247-253.

15. Larsen JV, Janowski K, Krolilowski A. Secondary post partum haemorrhage due to uterine wound dehiscence. Cent Afr J Med. 1995;41(9):294-296.

16. Pollio F, Staibano S, De Falco M, Buonocore U, De Rosa G, Di Lieto A. Severe secondary postpartum hemor- rhage 3 weeks after cesarean section: alternative etiologies of uterine scar non-union. J Obstet Gynaecol Res. 2007;33(3):360-362.

17. Tsai HF, Song HL, Chen WC, Chang CM, Chang CH, Lee IW. Delayed uterine rupture occurred 4 weeks after cesarean section following sexual intercourse: a case report and literature review. Taiwan J Obstet Gynecol. 2013;52(3):411-414.

18. Wagner MS, Bedard MJ. Postpartum uterine wound dehiscence: a case report. J Obstet Gynaecol Can. 2006;28(8):713-715.

19. Nanda S, Singhal S, Sharma D, Sood M, Singhal SK. Nonunion of uterine incision: a rare cause of secondary postpartum haemorrhage: a report of 2 cases. Aust N Z J Obstet Gynaecol. 1997;37(4):475-476.

20. Sumigama S, Sugiyama C, Kotani T, Hayakawa H, Inoue A, Mano Y, Tsuda H, et al. Uterine sutures at prior caesarean section and placenta accreta in subsequent pregnancy: a case-control study. BJOG. 2014;121(7):866-874; discussion 875.

21. Sholapurkar SL. Can the practice of nonclosure of visceral and parietal peritoneum during cesarean delivery be justified? Am J Obstet Gynecol. 2015;212(4):550.

22. The CAESAR study collaborative group. Caesarean section surgical techniques: a randomised factorial trial (CAESAR). BJOG. 2010;117(11):1366-1376.

23. Abalos E, Addo V, Brocklehurst P, El Sheikh M, Farrell B, Gray S, Hardy P, et al. Caesarean section surgical techniques (CORONIS): a fractional, factorial, unmasked, randomised controlled trial. Lancet. 2013;382(9888):234248.

24. Cheong YC, Premkumar G, Metwally M, Peacock JL, Li TC. To close or not to close? A systematic review and a meta-analysis of peritoneal non-closure and adhesion formation after caesarean section. Eur J Obstet Gynecol Reprod Biol. 2009;147(1):3-8.

25. Shi Z, Ma L, Yang Y, Wang H, Schreiber A, Li X, Tai $\mathrm{S}$, et al. Adhesion formation after previous caesarean section-a meta-analysis and systematic review. BJOG. 2011;118(4):410-422.

26. Kapustian V, Anteby EY, Gdalevich M, Shenhav S, Lavie $\mathrm{O}$, Gemer O. Effect of closure versus nonclosure of peritoneum at cesarean section on adhesions: a prospective randomized study. Am J Obstet Gynecol. 2012;206(1):56 e51-54.

27. El-Shawarby SA, Salim R, Lavery S, Saridogan E. Uterine adherence to anterior abdominal wall after caesarean section. BJOG. 2011;118(9):1133-1135.

28. Simmering J. Inter-ocular trauma test. http://jacobsimmering.com/2016/11/17/uhTickets/.

29. Friedman D, Sunder S. Statistical inference: preliminaries. In: Experimental Method - A Primer for Economists. 1994. Cambridge University Press, UK, Page 92-93. 